\title{
Biologia floral e da polinização de quatro espécies de Eugenia $\mathrm{L}$. (Myrtaceae)
}

\author{
André Luiz Gomes da Silva ${ }^{1,2}$ e Maria Célia B. Pinheiro ${ }^{1}$
}

Recebido em 12/01/2006. Aceito em 14/09/2006

\begin{abstract}
RESUMO - (Biologia floral e da polinização de quatro espécies de Eugenia L. (Myrtaceae)). Eugenia uniflora, E. punicifolia, E. neonitida e E. rotundifolia são espécies perenes, geralmente de porte arbustivo, que ocorrem na restinga do Parque Natural Municipal de Grumari, região oeste do Município do Rio de Janeiro. Possuem inflorescências racemosas com flores pediceladas inseridas nas axilas foliares. Suas flores são andróginas, polistêmones, do tipo Papaver, generalistas, com grãos de pólen como único recurso floral sendo enquadradas dentro da categoria de "flores-pólen". O estigma é seco, diminuto e formado por papilas delgadas. A antese é diurna, as flores duram apenas um dia e são visitadas por uma ampla variedade de insetos, incluindo himenópteros, dípteros, coleópteros e neurópteros, totalizando 29 espécies. As abelhas são os visitantes mais comuns e, dentre elas, Apis mellifera L., espécie introduzida pela ação humana, é a mais freqüente e abundante, sendo considerada o polinizador efetivo das espécies aqui estudadas. A floração é anual e em massa. E. uniflora floresce de agosto a outubro, com frutificação de setembro a novembro. E. neonitida floresce do final de agosto até início de dezembro, com frutificação de outubro até início de janeiro. E. punicifolia floresceu por duas vezes no ano de 2003, sendo a primeira florada entre os meses de junho e julho, frutificando no mês de agosto e a segunda, entre os meses de setembro e outubro, frutificando entre novembro e dezembro e no ano de 2004, floriu apenas no mês de agosto e frutificou entre outubro e dezembro. E. rotundifolia floresceu no mês de março, com frutificação de maio a junho.
\end{abstract}

Palavras-chave: Eugenia, Myrtaceae, restinga, biologia floral, polinização

ABSTRACT - (Floral and pollination biology of four species of Eugenia L. (Myrtaceae)). Eugenia uniflora, E. punicifolia, E. neonitida and $E$. rotundifolia are perennial species, usually shrubs, occurring in the restinga of Grumari Natural Municipal Park west of Rio de Janeiro. They have racemose inflorescences with pedicellate flowers inserted in the leaf axils. The flowers are androgynous, polystemonous, of the Papaver type, and generalists. Pollen grains are the only floral resource (classified as pollen-flowers). The stigma is dry, minute and made up of delicate papillae. Anthesis is diurnal. The flowers last only one day and are visited by a wide range of insects, including Hymenoptera, Diptera, Coleoptera and Neuroptera, for a total of 29 species. Bees are the most common visitors, with Apis mellifera L., a species introduced by human activities, the most frequent and abundant. This bee is regarded as the true pollinator of the species studied here. Flowering is yearly and massive. E. uniflora blooms from August to October, and fruits from September to November. E. neonitida blooms from late August to early December, and fruits from October to the first week in January. E. punicifolia bloomed twice in 2003, first in June and July, with fruiting in August, and second, from September to October, fruiting in November and December. In 2004, it bloomed only in August with fruiting from October to December. E. rotundifolia bloomed in March, with fruiting from May to June.

Key words: Eugenia, Myrtaceae, restinga, floral biology, pollination

\section{Introdução}

Myrtaceae é uma das maiores famílias botânicas com milhares de espécies reunidas em aproximadamente 140 gêneros, sendo importantes em diversos ecossistemas neotropicais (Johnson \& Briggs 1984; Landroum \& Kawasaki 1997; Wilson et al. 2001). Eugenia, com cerca de 1.000 espécies, é um dos maiores gêneros da família, com distribuição principalmente nas Américas Central e do Sul (Merwe et al. 2004) e está inserida na subfamília Myrtoideae, o qual inclui todos os gêneros de Myrtaceae que apresentam frutos carnosos (Lughadha \& Proença 1996).

A grande diversidade de espécies de Myrtaceae também se reflete nas restingas brasileiras (Assis et al. 2004) sendo a família mais representativa nas restingas do Estado do Rio de Janeiro e Eugenia, o gênero com

\footnotetext{
1 Universidade Federal do Rio de Janeiro, Programa de Pós-Graduação em Ciências Biológicas, Botânica do Museu Nacional, Quinta da Boa Vista, São Cristóvão, 20940-040 Rio de Janeiro, RJ, Brasil

2 Autor para correspondência: andrebotanico@gmail.com
} 
maior número de espécies (Araújo \& Henriques 1984; Lemos et al. 2001; Argôlo 2001). Na restinga de Grumari, Myrtaceae é representada por oito espécies e dois gêneros, na qual Eugenia é o mais representativo, com sete espécies (A.M. Argôlo, comentário pessoal).

O mecanismo reprodutivo de cada espécie é importante para assegurar a perpetuação de seus descendentes e para uma possível colonização de novos habitats, além de constituir a base para o desenvolvimento dos processos evolutivos naturais (Darwin 1859; Stebbins 1950; Grant 1971). Do ponto de vista econômico, a reprodução é um dos principais pilares para se manter uma cultura economicamente viável, seja ela através da produção de frutos e de sementes ou pela propagação vegetativa. Sendo assim, as análises sobre a biologia floral, o mecanismo de polinização e os registros fenológicos mostram-se de extrema importância, tanto para o meio natural quanto para a produção em escala comercial.

As espécies de Eugenia apresentam grãos de pólen como recurso floral, assim como muitas outras espécies de Myrtaceae (Faegri \& Pijl 1979; Proença \& Gibbs 1994; Lughadha \& Proença 1996). Os grãos de pólen são estruturas ricas em carboidratos, proteínas, vitaminas e lipídios e por esta razão, são importantes fontes alimentares para diversos visitantes florais (Faegri \& Pij1 1979; Free 1993; Roulston \& Cane 2000; Thorp 2000; Jones \& Jones 2001). Juntamente com outras espécies com flores pólen, como as poricidas, Eugenia desempenha um importante papel na manutenção deste recurso nas restingas (autor). No entanto, na família também há espécies nectaríferas (Peters \& Vasquez 1986/87) e outras em que partes do perianto são utilizadas como recurso alimentar por seus visitantes (Roitman et al. 1997).

A polinização nas Myrtaceae é muito diversificada e espécies desta família com flores não especializadas são visitadas por uma ampla variedade de animais, incluindo abelhas, vespas, moscas, pássaros e até mesmo mamíferos, sendo as abelhas os principais polinizadores (Beardsell et al. 1993; Proença \& Gibbs 1994; Lughadha \& Proença 1996). Espécies de abelhas da família Colletidae são os mais abundantes e importantes polinizadores de espécies australianas de Myrtaceae (Armstrong 1979) e em espécies africanas de Eugenia, as "abelhas de mel" Apidae - são os mais importantes visitantes (Wyk \& Lowrey 1988). Na região neotropical, Apidae é a família de abelhas mais comum entre as visitantes de Myrtaceae (Lughadha \& Proença 1996). Apis mellifera L., espécie de origem africana e mundialmente disseminada pela ação humana (Michener 1979), tem uma considerável interferência na biologia reprodutiva de muitas espécies vegetais podendo favorecer ou dificultar seu sucesso reprodutivo, além de influenciar direta ou indiretamente a dinâmica de forrageio de polinizadores nativos (Paton 1993; Vaughton 1996; Villanueva-G. 2002).

A fenologia reprodutiva é importante para os mecanismos de polinização, de predação e de dispersão de frutos e sementes, pois os aspectos temporais e de disponibilidade de recursos vegetais podem influenciar a dinâmica populacional de animais relacionados diretamente com estes mecanismos (Gleeson 1981; Ims 1990; Schaik et al. 1993; Nakagawa et al. 2003).

Estudos sobre a biologia reprodutiva de Myrtaceae, especialmente Eugenia, nas restingas do Brasil são escassos, embora se conheçam trabalhos sobre o mecanismo reprodutivo de espécies desta família em outros ecossistemas, destacando-se o de Proença \& Gibbs (1994) realizado na região central do Brasil, e o de Maués \& Couturier (2002) realizado na Amazônia paraense. Trabalhos importantes no contexto reprodutivo de Myrtaceae já foram realizados na África (Wyk \& Lowrey 1988) e na Austrália (Beardsell et al. 1993).

Nesse trabalho objetivou-se conhecer a biologia floral, o mecanismo de polinização e os aspectos fenológicos de Eugenia uniflora L., E. neonitida Sobral, E. punicifolia (Kunth) DC. e E. rotundifolia Cambess. na restinga do Parque Natural Municipal de Grumari.

\section{Material e métodos}

Área de estudo - Esta pesquisa foi realizada entre os anos de 2002 e 2004 na restinga de Grumari, localizada na região oeste do Município do Rio de Janeiro, nas

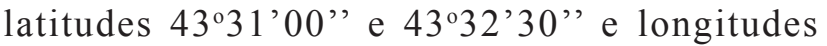
$23^{\circ} 02^{\prime} 30^{\prime \prime}$ e $23^{\circ} 03^{\prime} 10^{\prime \prime}$, entre os distritos do Recreio dos Bandeirantes e da Barra de Guaratiba. A restinga faz parte da APA de Grumari, que mais recentemente foi transformada em Parque Natural Municipal (DM 20149 de 2 de julho de 2001), e seu entorno está delimitado pelo Parque Estadual da Pedra Branca e pela APA da Prainha. Sua área total tem a forma de um imenso anfiteatro cercado pelo Maciço da Pedra Branca. Segundo A.M. Argôlo (comentário pessoal), há a formação de seis comunidades bem definidas nesta restinga, sendo elas: halófita, psamófila, pós-praia, arbustiva-aberta, arbustiva-fechada e mata de restinga. As espécies aqui em estudo são encontradas 
principalmente nas formações de pós praia e arbustivaaberta.

Espécies - Na restinga de Grumari, Myrtaceae é representada por oito espécies e dois gêneros, na qual Eugenia é o mais representativo, com sete espécies (A.M. Argôlo, comentário pessoal). E. uniflora possui porte arbustivo e pode alcançar até 5 metros de altura nesta restinga, no entanto, Legrand \& Klein (1969) relatam que esta espécie pode atingir até $12 \mathrm{~m}$ de altura. Dependendo da área onde se encontra, pode apresentar o aspecto de uma arvoreta ou arbusto com copa irregular quando dentro da mata, ou ainda de uma frondosa árvore, quando crescendo isoladamente. E. punicifolia tem aspecto arbustivo até arbóreo, podendo atingir até $3 \mathrm{~m}$ de altura, possui crescimento clonal e em uma mesma formação arbustiva pode-se encontrar de 10 a 50 ramos saindo do solo, oriundos de uma mesma ligação vegetativa. Eugenia neonitida é principalmente arbustiva podendo alcançar até $2,5 \mathrm{~m}$ de altura. E. rotundifolia é um arbusto que pode atingir até $3 \mathrm{~m}$ de altura e, assim como E. punicifolia, apresenta crescimento clonal, sendo encontradas formações arbustivas com até 30 ramos partindo do mesmo corpo vegetativo.

A biologia floral de cada espécie foi observada em populações naturais no campo e em flores e botões coletados e analisados no laboratório. Para análise em microscopia de varredura, os estiletes de flores recém abertas foram fixados em solução de gluteraldeído e posteriormente desidratados. A parte apical do estilete foi, então, fixada em um suporte de chumbo ("stub") e metalizada com ouro. As observações foram realizadas em microscópio Philips XL Series XL 20, S/W. ver. 5.21 .

A antese foi observada em um período de cinco dias para cada espécie, tendo sido averiguado o horário de abertura dos botões florais, a longevidade da flor, a receptividade do estigma e o processo de deiscência das anteras.

Para averiguação das estruturas florais responsáveis pela liberação do odor, foi feito o teste com vermelho neutro, que indica a presença de atividade metabólica nos tecidos corados (Vogel 1990).

Foram realizadas medições do comprimento do estilete de botões em pré-antese e naqueles de flores recém abertas, para se verificar a possibilidade de deposição de grãos de pólen da própria flor em seu estigma durante o processo de abertura floral.

Polinização - As observações dos visitantes florais foram realizadas durante os episódios de pico de floração de Eugenia uniflora, E. punicifolia, E. rotundifolia e E. neonitida entre os anos de 2003 e 2004. Para cada espécie foram anotados os horários de visitas, o comportamento intrafloral, intraplanta e interplanta e o contato entre o corpo do animal e os verticílos reprodutores.

Nas quatro espécies aqui estudas foram realizadas observações dos visitantes florais durante os respectivos períodos de floração em massa. De cada espécie de planta foi escolhido um indivíduo focal de onde foram anotados os horários de visitas discriminadas de cada grupo de inseto, abelhas, moscas e besouros. Destas observações foi calculada uma média do número de visitas nas quatro espécies entre $5 \mathrm{~h} 30 \mathrm{~min}$ e $12 \mathrm{~h}$. Apis mellifera L. foi avaliada separadamente em virtude de sua importância na biologia reprodutiva destas espécies.

Todos os insetos observados durante as visitas florais foram coletados com saco plástico ou rede entomológica e posteriormente encaminhados a especialistas para determinação taxonômica.

Fenologia - A fenologia reprodutiva foi verificada através das observações realizadas em cinco plantas marcadas de cada espécie entre os anos de 2003 e 2004. Foram anotadas as épocas de início de emissões florais, a duração da floração e o início e a duração do período de frutificação de cada espécie.

\section{Resultados e discussão}

Biologia floral - Eugenia uniflora, E. punicifolia, E. rotundifolia e E. neonitida apresentam inflorescências racemosas com flores pediceladas inseridas nas axilas foliares. E. neonitida emite uma única flor por cada axila foliar, porém, como as folhas das Myrtaceaes são opostas, de cada nó foliar são emitidas duas flores. E. uniflora, E. punicifolia, E. rotundifolia apresentam um padrão diferenciado, pois de cada nó foliar são emitidas mais de duas flores (Tab. 1). Segundo Briggs \& Johnson (1979), o gênero Eugenia é caracterizado por apresentar uniflorescências do tipo conflorescência racemosa mônade, derivada de regressões sucessivas da inflorescência paniculiforme ao longo da linha evolutiva das espécies de Myrtaceae. Segundo estes mesmos autores, algumas espécies de Eugenia possuem uma agregação funcional de conflorescências que são denominadas de superconflorescências, como é o caso de E. uniflora, E. punicifolia, E. rotundifolia.

E. uniflora, E. neonitida, E. punicifolia e 
Tabela 1. Estruturas florais de espécies de Eugenia.

\begin{tabular}{|c|c|c|c|c|}
\hline & Eugenia uniflora & Eugenia punicifolia & Eugenia neonitida & Eugenia rotundifolia \\
\hline Diâmetro da flor (mm) & $\begin{array}{c}16,1 \pm 1,2 \\
\mathrm{~N}=10\end{array}$ & $\begin{array}{c}14,5 \pm 1,6 \\
\mathrm{~N}=15\end{array}$ & $\begin{array}{c}25,7 \pm 2,6 \\
\mathrm{~N}=20\end{array}$ & $\begin{array}{c}14,9 \pm 1,48 \\
\mathrm{~N}=20\end{array}$ \\
\hline Diâmetro do estigma (mm) & $\begin{array}{c}0,24 \pm 0,05 \\
\mathrm{~N}=15\end{array}$ & $\begin{array}{c}0,20 \pm 0,03 \\
\mathrm{~N}=15\end{array}$ & $\begin{array}{c}0,23 \pm 0,02 \\
\mathrm{~N}=15\end{array}$ & $\begin{array}{c}0,15 \pm 0,37 \\
\mathrm{~N}=25\end{array}$ \\
\hline Diâmetro do ovário (mm) & $\begin{array}{c}1,2 \pm 0,1 \\
\mathrm{~N}=20\end{array}$ & $\begin{array}{c}1,2 \pm 0,1 \\
\mathrm{~N}=20\end{array}$ & $\begin{array}{c}2,3 \pm 0,4 \\
\mathrm{~N}=20\end{array}$ & $\begin{array}{c}2,11 \pm 0,16 \\
\mathrm{~N}=20\end{array}$ \\
\hline Óvulos/ovário & $\begin{array}{c}20,8 \pm 3,5 \\
\mathrm{~N}=20\end{array}$ & $\begin{array}{c}28,5 \pm 6,2 \\
\mathrm{~N}=20\end{array}$ & $\begin{array}{c}33,8 \pm 5,6 \\
\mathrm{~N}=20\end{array}$ & $\begin{array}{c}20,8 \pm 2,9 \\
\mathrm{~N}=20\end{array}$ \\
\hline Comprimento do estilete $(\mathrm{mm})$ & $\begin{array}{c}6,08 \pm 0,9 \\
\mathrm{~N}=26\end{array}$ & $\begin{array}{c}5,5 \pm 1,2 \\
\mathrm{~N}=22\end{array}$ & $\begin{array}{c}7,8 \pm 0,8 \\
\mathrm{~N}=15\end{array}$ & $\begin{array}{c}9,5 \pm 0,61 \\
\mathrm{~N}=20\end{array}$ \\
\hline Número de estames por flor & $\begin{array}{c}52,9 \pm 6,9 \\
\mathrm{~N}=15\end{array}$ & $\begin{array}{c}42,4 \pm 7,1 \\
\mathrm{~N}=20\end{array}$ & $\begin{array}{c}143,7 \pm 26 \\
N=10\end{array}$ & $\begin{array}{c}84,2 \pm 11,17 \\
\mathrm{~N}=15\end{array}$ \\
\hline Flor/axila foliar & $\begin{array}{c}3,3 \pm 0,9 \\
\mathrm{~N}=20\end{array}$ & $\begin{array}{c}3,5 \pm 2,4 \\
\mathrm{~N}=20\end{array}$ & $\begin{array}{c}1,0 \pm 0 \\
\mathrm{~N}=20\end{array}$ & $\begin{array}{c}3,05 \pm 2,04 \\
\mathrm{~N}=20\end{array}$ \\
\hline
\end{tabular}

E. rotundifolia apresentam de quatro a sete nós foliares por ramo vegetativo e, durante o pico de floração, as flores se destacam da folhagem dando um aspecto branco às plantas, assim como ocorre em muitas outras espécies de Myrtaceae (Briggs \& Johnson 1979; Beardsell et al. 1993; Lughadha \& Proença 1996). Esta agregação de flores é o que caracteriza a floração em massa (Gentry 1974), e pode ser considerada como uma estratégia para atração dos polinizadores (Faegri \& Pijl 1979; Bawa 1983; O’Brien \& Calder 1993).

O início da antese se dá logo ao amanhecer, por volta das $5 \mathrm{~h} 30 \mathrm{~min}$ e se estende durante todo o dia. A antese nas quatro espécies é do tipo Psidium, pois os filamentos estaminais e o estilete se espandem juntamente com o desabrochar das pétalas durante a abertura floral (Proença \& Gibbs 1994). Durante o período de antese, os grãos de pólen das espécies aqui estudadas estão totalmente expostos, não havendo assim, quaisquer restrições a sua coleta, característica esta comum das flores generalistas (Faegri \& Pij1 1979; Endress 1994).

As flores das quatro espécies são hermafroditas, com quatro pétalas alvas curvadas sobre o hipanto e quatro sépalas também flexionadas sobre esta estrutura, proporcionando um maior destaque ao androceu (Fig. 1). O androceu é polistêmone com muitos estames, variando de 42,4 em Eugenia punicifolia até $143,7 \mathrm{em} E$. neonitida. Os estames apresentam filetes filiformes e anteras globosas, bitecas e de deiscência longitudinal. Em E. uniflora as anteras são de coloração amarelada, e em E. neonitida, E. punicifolia, E. rotundifolia de coloração branca.
Os estames estão distribuídos sobre o hipanto ao redor do estilete e o seu número é variável entre as quatro espécies (Tab. 1). Este elevado número de estames produz uma grande quantidade de grãos de pólen que funcionam como atrativo floral primário para os visitantes florais (Faegri \& Pijl 1979). De fato, esta é uma característica comum entre as espécies de Eugenia (Wyk \& Lowrey 1988; Proença \& Gibbs 1994) e de outras Myrtoideae (Lughadha \& Proença 1996). Segundo Beardsell et al. (1993), o número de estames pode variar de cinco até 300 em espécies australianas de Myrtaceae.

As flores de Eugenia uniflora, E. punicifolia, E. rotundifolia e E. neonitida são denominadas de "flores-pólen" pelo fato do pólen ser o único recurso floral oferecido aos polinizadores (Faegri \& Pij1 1979; Endress 1994). São enquadradas também no tipo Papaver, segundo a classificação proposta por Vogel (1978). Faegri \& Pijl (l.c.), Free (1993), Roulston \& Cane (2000) e Thorp (2000) relataram que os grãos de pólen são fontes ricas em nitrogênio/proteína, vitaminas, carboidratos e lipídios onde são utilizados, principalmente, como alimento para as larvas de abelhas, moscas, besouros, vespas, pássaros e morcegos.

As flores das quatro espécies apresentam ovário ínfero, bilocular e pluriovulado, com o número de óvulos variando entre elas (Tab. 1). O estigma é diminuto, seco e formado por papilas (Fig. 2) o que parece ser um padrão comum entre as espécies de Myrtoideae (Wyk \& Lowrey 1988; Proença \& Gibbs 1994; Lughadha \& Proença 1996), embora outras espécies de Myrtaceae possam apresentar estigma úmido 
(Heslop-Harrison \& Shivanna 1977; Beardsell et al. 1993; Proença \& Gibbs 1994). O diâmetro do estigma variou pouco entre as espécies aqui estudadas (Tab. 1). Durante o processo de abertura floral o estigma já se encontra receptivo, pois neste momento suas papilas estão túrgidas e brilhosas.

O estilete é ereto, porém sua extremidade apical, abaixo do estigma, pode apresentar uma leve curvatura. No início da antese, esta curvatura não é evidente, se acentuando ao longo do dia. No estilete de E. neonitida e E. rotundifolia foi evidenciado, ao microscópico óptico, estruturas glandulares, que nas outras duas espécies não foram encontradas.
O crescimento em comprimento do estilete ocorre durante a maturação do botão em pré-antese e, no início da antese ainda não alcançou seu tamanho máximo. Em análises de estiletes na fase de botão 24 horas antes de sua abertura e em flores recém abertas, verificou-se uma diferença significativa no comprimento destes, com aumento de tamanho ultrapassando 50\% (Tab. 2). Este crescimento contínuo do estilete durante a abertura floral promove a adesão de grãos de pólen ao estigma, pois nesta fase as anteras já se encontram deiscentes, o que parece ser uma característica comum em algumas espécies de Myrtaceae (Crome \& Irvine 1986; Beardsell et al. 1993; Proença \& Gibbs 1994). Nas
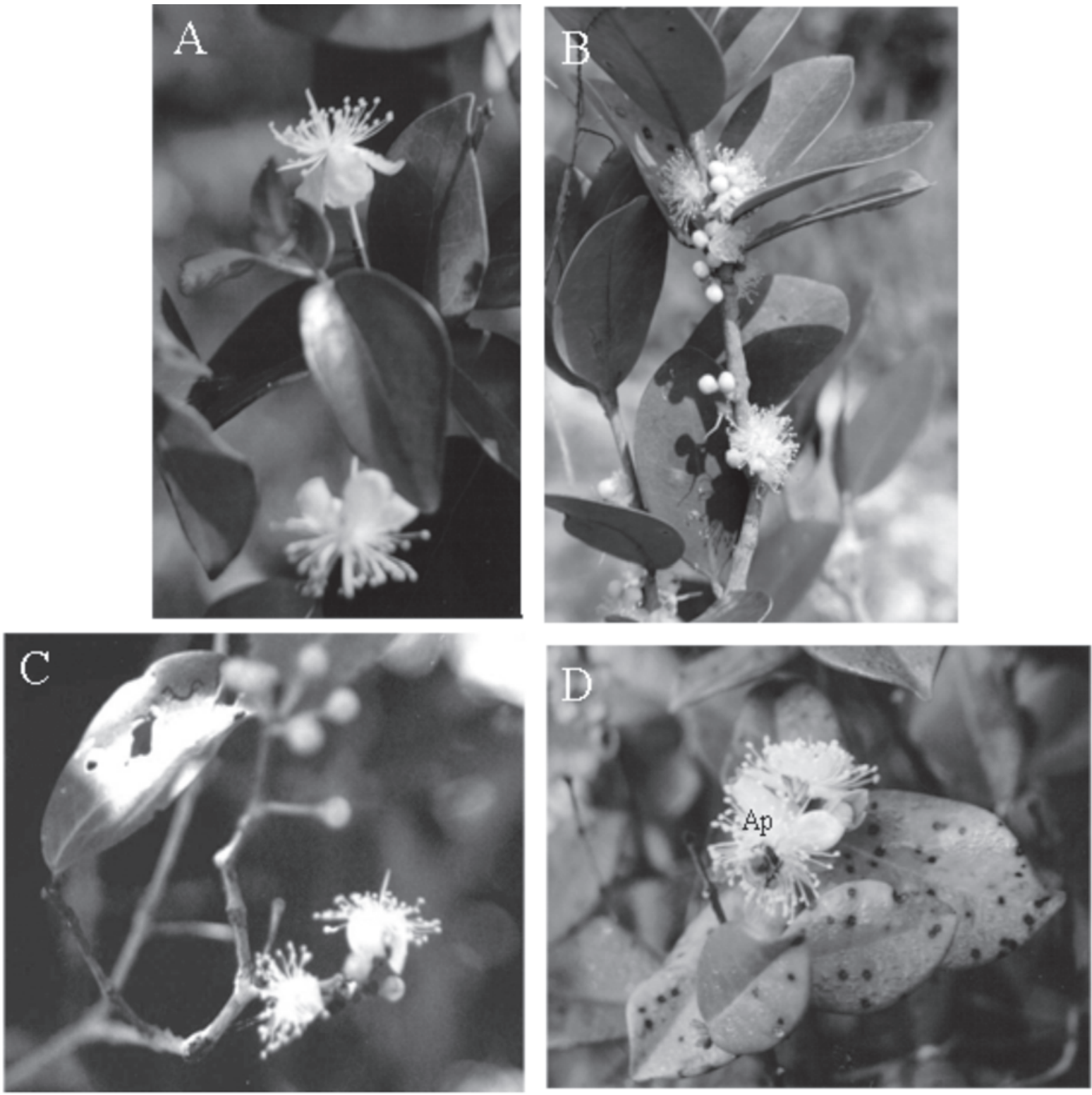

Figura 1. Ramos floridos: A. Eugenia uniflora L. B. E. rotundifolia Cambess. C. E. punicifolia (Kunth) DC.; D. E. neonitida Sobral. Ap - Apis mellifera L. visitando uma flor de E. neonitida. 

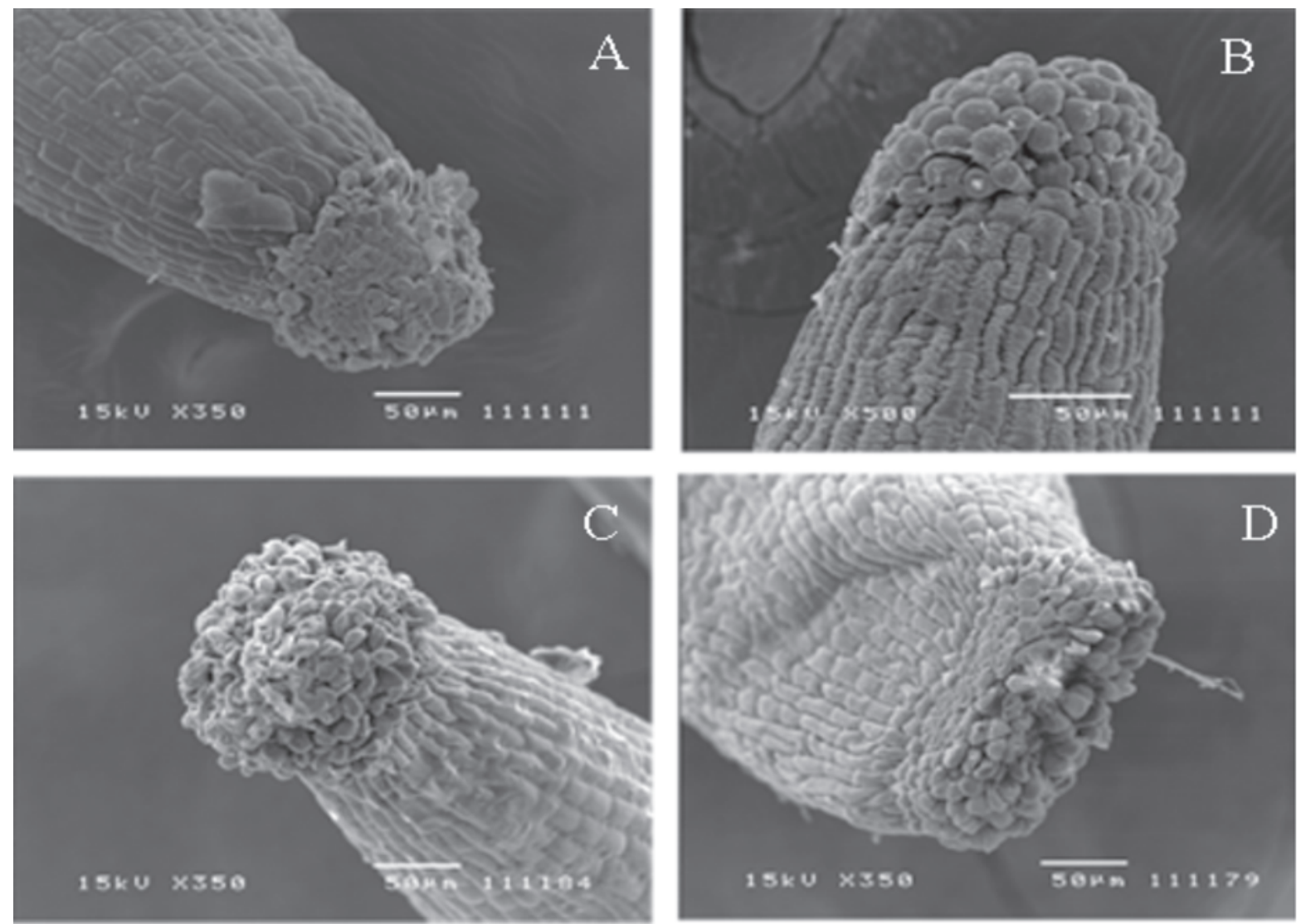

Figura 2. Estigmas papilosos vistos em microscopia de varredura: A. Eugenia uniflora L. B. E. rotundifolia Cambess. C. E. punicifolia (Kunth) DC. D. E. neonitida Sobral.

espécies aqui estudadas a análise de estigmas de flores ensacadas na fase de botões e, conseqüentemente, excluídas de polinizadores mostrou uma quantidade significativa de grãos sobre suas papilas estigmáticas podendo assim promover a autopolinização automática (dados não publicados).

Nas primeiras horas da manhã, as flores exalam um odor suave e adocicado e cada espécie possui uma

Tabela 2. Comprimento do estilete $(\mathrm{mm})$ de botões em pré-antese (24 horas antes da abertura floral) e de flores recém abertas de espécies de Eugenia.

\begin{tabular}{lccc}
\hline & $\begin{array}{c}\text { Estilete no } \\
\text { botão }\end{array}$ & $\begin{array}{c}\text { Estilete na } \\
\text { flor }\end{array}$ & $\begin{array}{c}\text { Crescimento } \\
\text { total }\end{array}$ \\
\hline Eugenia uniflora & $3,3 \pm 0,8$ & $6,08 \pm 0,9$ & $84 \%$ \\
& $\mathrm{~N}=15$ & $\mathrm{~N}=26$ & \\
Eugenia uniflora & $4,85 \pm 1,3$ & $7,8 \pm 0,8$ & $61 \%$ \\
& $\mathrm{~N}=10$ & $\mathrm{~N}=15$ & \\
Eugenia uniflora & $6,05 \pm 0,76$ & $9,5 \pm 0,61$ & $57 \%$ \\
& $\mathrm{~N}=20$ & $\mathrm{~N}=20$ & \\
Eugenia uniflora & $3,45 \pm 0,4$ & $5,5 \pm 1,2$ & $59 \%$ \\
& $\mathrm{~N}=15$ & $\mathrm{~N}=22$ & \\
\hline
\end{tabular}

fragrância específica, sendo facilmente distinguíveis no campo. Em testes realizados com vermelho neutro, apenas as anteras se coram, indicando que estas estruturas podem ser as responsáveis pela emissão do odor. Wyk \& Lowrey (1988), estudando espécies de Eugenia da África do Sul, observaram que o adocicado odor das flores era emitido pelos tecidos das anteras, ou pelas glândulas do conectivo das anteras ou então pelos grãos de pólen, características comuns a outras espécies vegetais não correlatas (Dobson \& Bergström 2000).

Dreller \& Tarpy (2002) e Pernal \& Currie (2002) ressaltaram que determinadas espécies de abelhas são atraídas, principalmente, pelo odor liberado dos óleos voláteis encontrados nos grãos de pólen em famílias não correlacionadas. De fato, a liberação de odor parece ser uma característica marcante nas Myrtaceae (Wyk \& Lowrey 1988; Proença \& Gibbs 1994; Maués \& Couturier 2002) e está diretamente ligada ao mecanismo de polinização (Faegri \& Pijl 1979; Endress, 1994).

As flores permanecem receptivas até parte da tarde e no segundo dia, as pétalas e o androceu caem, 
persistindo apenas o cálice e o estilete, que perduram ainda nos frutos jovens. Estas características são comuns a outras espécies de Eugenia (Wyk \& Lowrey 1988; Proença \& Gibbs 1994) além de outras Myrtoideae (Lughadha \& Proença 1996).

Polinização - As flores de E. uniflora, E. neonitida, E. punicifolia e E. rotundifolia foram visitadas por um total de 29 espécies de insetos (Tab. 3), das quais 15 eram de himenópteros $(51,7 \%)$, oito de coleópteros $(27,6 \%)$, cinco de dípteros $(17,2 \%)$ e uma de neoróptero (3,4\%) (Fig. 3). Deste total, 19 (65,5\%) espécies foram observadas em E. uniflora, $12(41,4 \%)$ em E. neonitida, $18(62,1 \%)$ em E. punicifolia e nove (31\%) em E. rotundifolia (Fig. 4). Dentre todos os insetos observados e coletados, as abelhas foram os visitantes mais comuns, exceto em E. neonitida onde o número de espécies de himenópteros observados foi igual ao número de espécies de coleópteros, cinco no total (Fig. 5).

Muitas outras espécies de Myrtaceae com flores generalistas também são visitadas por um diversificado grupo de animais, incluindo insetos, morcegos e pássaros (O’Brien \& Calder 1993; Beardsell et al. 1993; Proença \& Gibbs 1994; Lughadha \& Proença 1996; Ducroquet \& Hickel 1997; Degenhardt et al. 2001). No entanto, Armstrong (1979), Peters \& Vasquez (1986/87), Wyk \& Lowrey (1988), Beardsell et al. (1993), Proença \& Gibbs (1994) e Lughadha \& Proença (1996) ressaltam que as abelhas são seus visitantes mais comuns.

Nas espécies aqui estudadas, os visitantes florais mais abundantes e freqüentes foram as operárias de Apis mellifera (Fig. 2D). Os indivíduos desta espécie iniciam seu forrageio logo ao amanhecer, por volta das

Tabela 3. Visitantes florais de espécies de Eugenia.

\begin{tabular}{|c|c|c|c|c|}
\hline & $\begin{array}{c}\text { Eugenia } \\
\text { uniflora }\end{array}$ & $\begin{array}{c}\text { Eugenia } \\
\text { punicifolia }\end{array}$ & $\begin{array}{l}\text { Eugenia } \\
\text { neonitida }\end{array}$ & $\begin{array}{l}\text { Eugenia } \\
\text { rotundifolia }\end{array}$ \\
\hline \multicolumn{5}{|l|}{ Diptera } \\
\hline Allograpta sp. (Syrphidae) & $\mathrm{X}$ & & $\mathrm{X}$ & $\mathrm{X}$ \\
\hline Ocyptamus sp. (Syrphidae) & & & $\mathrm{X}$ & \\
\hline Ornidia obesa Lepeletier \& Serville, 1828 (Syrphidae) & $\mathrm{X}$ & $\mathrm{X}$ & $\mathrm{X}$ & $\mathrm{X}$ \\
\hline Tachinidae Tribo Goniini - sp. 1 & & & $\mathrm{X}$ & \\
\hline Tephritidae - sp. 2 & $\mathrm{X}$ & $\mathrm{X}$ & $\mathrm{X}$ & $\mathrm{X}$ \\
\hline \multicolumn{5}{|l|}{ Neoroptera } \\
\hline sp. 1 & $\mathrm{X}$ & & & \\
\hline \multicolumn{5}{|l|}{ Coleoptera } \\
\hline Nitidulidae & $\mathrm{X}$ & & $\mathrm{X}$ & \\
\hline Eliateridae & & & $\mathrm{X}$ & \\
\hline Chrysomelidae (Cristocephalinae) & & $\mathrm{X}$ & $\mathrm{X}$ & \\
\hline Oedemeridae & $\mathrm{X}$ & $\mathrm{X}$ & $\mathrm{X}$ & $\mathrm{X}$ \\
\hline Astylus lineatus Fabricius, 1775 (Dasitidae) & $\mathrm{X}$ & $\mathrm{X}$ & $\mathrm{X}$ & $\mathrm{X}$ \\
\hline Teneobrionidae & & $\mathrm{X}$ & & \\
\hline Scarabaeidae (Melolothinae) & & $\mathrm{X}$ & & \\
\hline Coccinellidae & $\mathrm{X}$ & & & \\
\hline \multicolumn{5}{|l|}{ Hymenoptera } \\
\hline Exomalopsis analis Spinola, 1853 (Apidae) & & & $\mathrm{X}$ & \\
\hline Melípona bicolor Lepeletier, 1836 (Apidae) & & & $\mathrm{X}$ & \\
\hline Melipona quadrifasciata Lepeletier, 1836 (Apidae) & & & $\mathrm{X}$ & \\
\hline Apis mellifera Linnaeus, 1758 (Apidae) & $\mathrm{X}$ & $\mathrm{X}$ & $\mathrm{X}$ & $\mathrm{X}$ \\
\hline Scaptotrigona xanthotricha Moure, 1950 (Apidae) & $\mathrm{X}$ & & $\mathrm{X}$ & \\
\hline Exomalopsis analis Spinola, 1853 (Apidae) & $\mathrm{X}$ & & & \\
\hline Trigona spinipes Fabricius, 1793 (Apidae) & $\mathrm{X}$ & & & \\
\hline Partamona helleri Friese, 1900 (Apidae) & $\mathrm{X}$ & $\mathrm{X}$ & $\mathrm{X}$ & $\mathrm{X}$ \\
\hline Xylocopa sp. (Apidae) & $\mathrm{X}$ & & & \\
\hline Centris sp. (Apidae) & $\mathrm{X}$ & & & \\
\hline Augochlora sp. (Halictidae) & & $\mathrm{X}$ & & \\
\hline Augochloropsis melanochaeta Moure, 1950 (Halictidae) & $\mathrm{X}$ & & & \\
\hline Dialictus sp. 1 (Halictidae) & $\mathrm{X}$ & & & \\
\hline Dialictus sp. 2 (Halictidae) & $\mathrm{X}$ & $\mathrm{X}$ & $\mathrm{X}$ & $\mathrm{X}$ \\
\hline Ptiloglossa sp. (Colletidae) & $\mathrm{X}$ & $\mathrm{X}$ & $\mathrm{X}$ & $\mathrm{X}$ \\
\hline
\end{tabular}




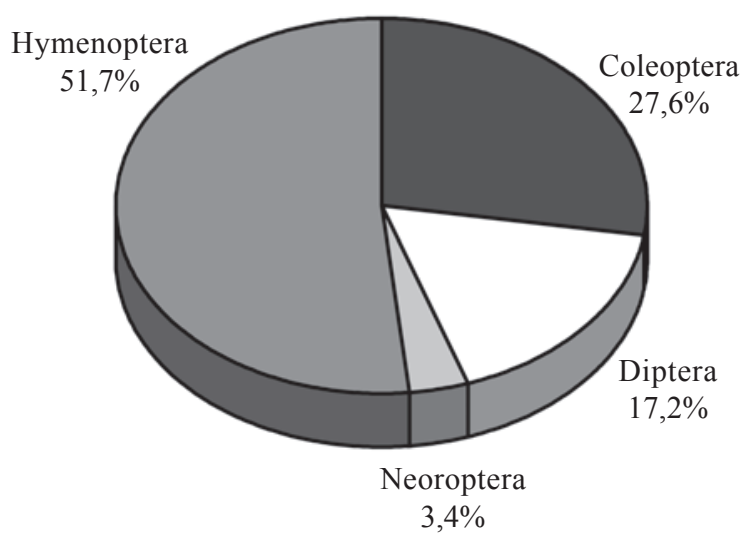

Figura 3. Proporções entre as ordens de visitantes florais de Eugenia uniflora L, E. neonitida Sobral, E. punicifolia (Kunth) DC. e E. rotundifolia Cambess.

$5 \mathrm{~h} 30 \mathrm{~min}$, e entre $6 \mathrm{~h}$ e $7 \mathrm{~h}$ há um pico de atividades de visitas (Fig. 5). Em E. punicifolia, E. uniflora e E. rotundifolia foram contados até cerca de 50 operárias forrageando juntas uma mesma planta. Por outro lado, em E. neonitida, em que a densidade floral é menor, foi contado um número máximo de vinte abelhas em uma mesma planta durante o pico de floração. Em dias claros, ensolarados e com baixa umidade relativa do ar, em torno das sete horas da manhã, as anteras já estão completamente esvaziadas de grãos de pólen em decorrência das diversas visitas recebidas, fato também comum a outras espécies com recurso pólen (Peters \& Vasquez 1986/1987; Maués \& Couturier 2002; Silva et al. 2002). A intensidade de visitas de $A$. mellifera no início da antese pode prejudicar o suprimento de grãos de pólen para os visitantes nativos e, desta forma, estas espécies tendem a visitar um número maior de flores para conseguir um suprimento adequado de alimento. Segundo Paton (1993), o desprendimento maior de energia para coleta de pólen pode interferir negativamente no sucesso reprodutivo das espécies de visitantes nativos.

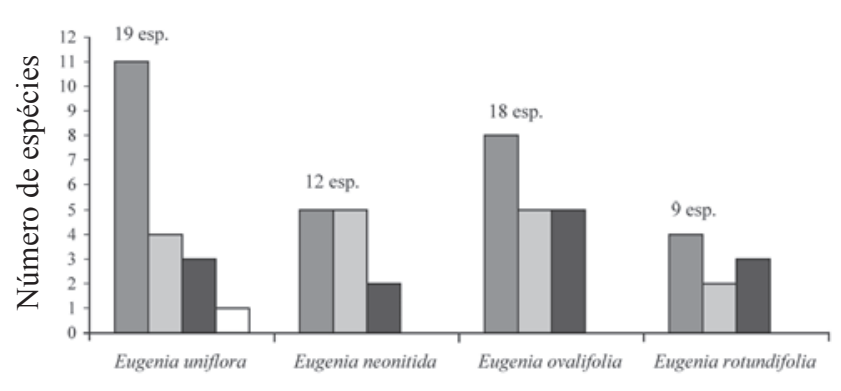

Figura 4. Visitantes florais observados em espécies de Eugenia. Números à cima das barras representam o total de espécies de insetos observados em cada espécie vegetal ( $\square=$ Hymenoptera; $\square=$ Coleoptera; $\square=$ Diptera; $\square=$ Neoroptera $)$.
Durante as visitas de $A$. mellifera às flores de E. uniflora, E. neonitida, E. punicifolia e E. rotundifolia, é pouco comum à presença de outras espécies de visitantes florais, o que nos permite inferir que esta abelha, em função de seu comportamento agressivo, pode limitar o comportamento forrageiro de espécies nativas, o que também já foi observado por Taylor \& Whelan (1988), Paton (1993) e Aguiar \& Martins (2003), para outras espécies vegetais.

A. mellifera visita uma grande variedade de flores, independentemente de atuar como polinizadora ou não (Hopper 1980; Taylor \& Whelan 1988; Ramalho et al. 1990; Paton 1993; Proença \& Gibbs 1994; Vaughton 1996; Huryn 1997; Gross \& Mackay 1998; Moço \& Pinheiro 1999; Villanueva-G. 2002; Maués \& Couturier 2002; Siqueira de Castro 2002; Lorenzon et al. 2003; Anderson 2003; Ramalho 2003). Marchini et al. (2001), em um estudo realizado em duas localidades de São Paulo, encontraram 157 espécies de plantas visitadas por A. mellifera, sendo quatro de Eugenia, incluindo Eugenia uniflora. Em espécies de Eugenia cultivadas da Bahia, dentre elas também E. uniflora, Siqueira de Castro (2002) afirma que A. mellifera foi um dos principais visitantes florais. Lorenzon et al. (2003) relatam que esta espécie de abelha possui um comportamento generalista e que é importante para grande parte da flora da caatinga do Parque Nacional da Capivara, PI, Brasil.

Embora Apis mellifera seja uma espécie de grande potencial econômico, com importância na produção de mel, de seus derivados e na polinização de cultivares agrícolas (Free 1993; Huryn 1997; Lorenzon et al. 2003) não há nenhum estudo de impacto ambiental ocasionado por estas abelhas nas restingas

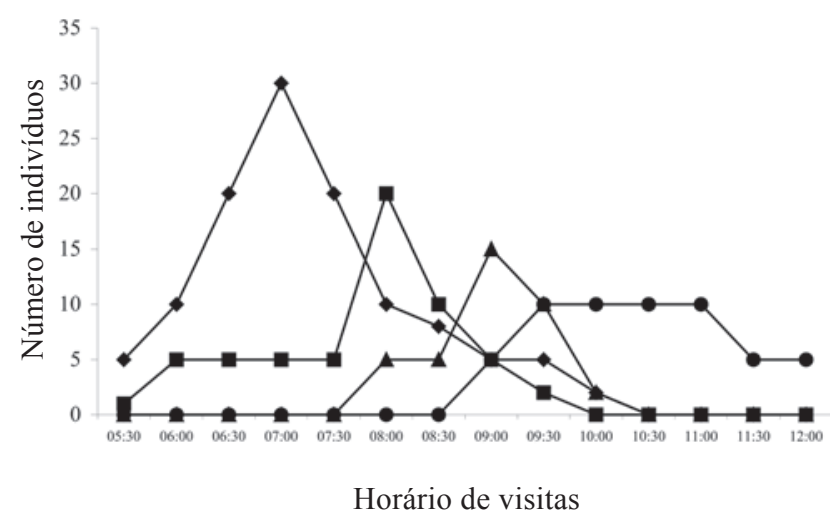

Figuras 5. Número de visitantes florais observados ao longo de três dias consecutivos em uma planta focal de cada espécie: Eugenia uniflora L, E. neonitida Sobral, E. punicifolia (Kunth) DC. e E. rotundifolia Cambess. $(--=$ Apis mellifera; $-\mathbf{-}=$ Outras abelhas; $-\mathbf{\Delta}-=$ Moscas; $-\bullet-=$ Besouros $)$. 
brasileiras, sendo necessárias pesquisas nesta área a fim de avaliar as reais interações com as plantas por elas visitadas e com as espécies de polinizadores nativos.

Outras quatorze espécies de abelhas visitam as flores de Eugenia uniflora, E. neonitida, E. punicifolia e E. rotundifolia. A família Apidae é a melhor representada com oito espécies, seguida de Halictidae com quatro espécies, Colletidae com uma espécie e Anthophoridae com duas espécies (Fig. 6). Lughadha \& Proença (1996); Siqueira de Castro (2002); Ramalho (2003) e Aguiar \& Martins (2003) constataram que, de fato, Apidae é a família de abelhas mais importante da região neotropical, seguida de Halictidae.

O tempo de permanência destas abelhas nas flores das espécies aqui estudadas é o mesmo de Apis mellifera, cerca de três a cinco segundos, porém o tempo de forrageio entre as plantas é bem menor. Xylocopa sp., abelha solitária e de grande porte, foi observada, nas quatro espécies estudadas, em uma freqüência muito baixa, cerca de uma visita por dia. Em seu vôo forrageiro, esta abelha pousa em uma flor, permanece nela durante cerca de quatro segundos e, em seguida, parte sem realizar outras visitas, comportamento observado nas quatro espécies aqui estudadas. O mesmo ocorreu com indivíduos de Centris sp., abelha também solitária e de médio porte. Embora os indivíduos destas espécies contatem o androceu e o estigma durante as visitas, é muito improvável que realizem a polinização, em virtude de sua baixa freqüência de visitas. No entanto, Bombus spp., abelha também de médio porte, foi considerada por Proença (1992) e Proença \& Gibbs (1994), o principal grupo polinizador de Eugenia dysenterica Mart. ex DC., entre outras espécies de Myrtaceae ocorrentes na região central do Brasil.

Abelhas nativas de pequeno porte como Trigona spinipes Fabricius, Augochloropsis melanochaeta Moure, Melipona quadrifasciata Lepeletier, entre outras (Tab. 3), também foram observadas neste estudo, porém com freqüência de visitas inferior a de Apis mellifera e, dentre estas, T. spinipes é a visitante mais comum. Todas estas abelhas iniciam suas atividades forrageiras após o pico de visitas de A. mellifera, sendo assim, pouco provável de realizarem a polinização (Fig. 6).

Cinco espécies de dípteros foram observadas, sendo três da família Syrphidae, uma de Tachinidae e outra de Tephritidae. Entre elas Ornidia obesa Lepeletier \& Serville 1828 (Syrphidae) foi a mais

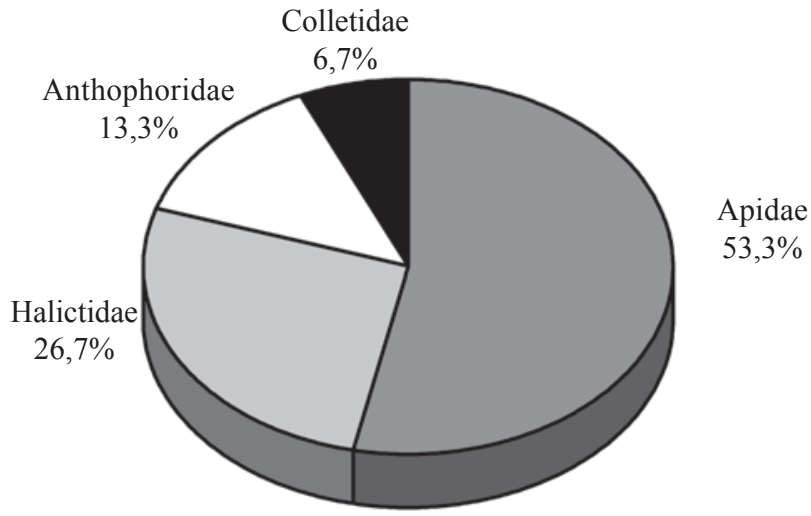

Figura 6. Proporções entre as famílias de abelhas observadas nas flores de Eugenia uniflora L, E. neonitida Sobral, E. punicifolia (Kunth) DC. e E. rotundifolia Cambess.

freqüente e abundante. $\mathrm{O}$ comportamento intrafloral e de forrageio destas espécies de moscas são similares entre si, visitando várias flores por vôo e permanecendo nas flores por cerca de três a cinco segundos e, durante estas visitas, as anteras e o estigma são contatados. Suas atividades forrageiras ocorrem principalmente no horário da manhã, com maior incidência de visitas no período entre $08 \mathrm{~h}$ e $10 \mathrm{~h}$, horário que não coincide com o pico de atividade de Apis mellifera (Fig. 6).

Segundo Proctor \& Yeo (1973) e Larson et al. (2001) os dípteros são, entre todos os insetos do mundo, o segundo maior grupo de visitantes florais e, dentre eles, os sirfídios são os mais comuns em muitas espécies vegetais não correlatas (Machado \& Loiola 2000; Machado \& de Oliveira 2000; Larson et al. 2001), assim como em espécies de Myrtaceae (Maués $\&$ Couturier 2002). É pouco provável que estes insetos realizem a polinização nas espécies aqui estudadas, pelo fato das abelhas, principalmente A. mellifera, visitarem intensamente as flores no início da antese. Segundo Sazima (1978); Proctor \& Yeo (1973) e Larson et al. (2001) as espécies de dípteros podem atuar tanto como polinizadores quanto pilhadores dos recursos florais.

Oito espécies de coleópteros visitaram as flores das espécies aqui estudadas e, de todos estes, Astylus lineatus Fabricius (Dasitidae) foi o besouro mais freqüente e abundante. Durante as visitas, estes insetos permaneciam por muito tempo, cerca de dez minutos, em cada flor, e não contatam a região estigmática. Suas atividades ocorreram, principalmente, a partir das 9 horas da manhã (Fig. 5) e neste horário, a quantidade de grãos de pólen disponível nas anteras das quatro espécies era muito pequena ou quase nula e, por esta razão, pouquíssimos grãos foram encontrados em seu corpo. Estes insetos pilhavam os grãos de pólen caídos 
sobre o perianto ou então, consomiam parte das estruturas florais.

Os besouros, de uma forma geral, não são eficientes polinizadores de espécies de Eugenia, pois sua morfologia e seu comportamento intrafloral e interplanta não permitem uma eficiente transferência de grãos de pólen (Faegri \& Pijl 1979; O’Brien \& Calder 1993; Endress 1994). A polinização por besouros pode ser comum em algumas outras espécies de Myrtaceae (Beardsell et al. 1993) e segundo Maués \& Couturier (2002) os coleópteros atuam como polinizadores ocasionais ou ilegítimos de Myrciaria dubia (H.B.K.) McVaugh. Em Eugenia uniflora, foi observada uma espécie de Neuroptera visitando as flores, embora as espécies que integram esta ordem não sejam associadas à polinização (Anderson 2003).

Os principais visitantes florais de Eugenia uniflora, E. neonitida, E. punicifolia e $E$. rotundifolia, que são abelhas de pequeno porte e moscas, apresentam uma amplitude forrageira baixa, comportamento que pode vir a diminuir o fluxo gênico entre as populações vegetais, diferentemente do que acontece com grandes polinizadores, como abelhas de grande porte, pássaros e mamíferos que podem carregar o pólen por longas distâncias (Collevatti et al. 2000; Latouche-Hallé et al. 2004).

De todos os visitantes florais observados, Apis mellifera mostrou ser o principal polinizador das espécies aqui estudadas. Esta constatação se deve, principalmente, ao fato de que os indivíduos desta espécie são os primeiros visitantes florais no início da antese e que, durante as visitas, o corpo destas abelhas contata tanto o androceu quanto o estigma. Embora A. mellifera possa ser considerada uma ameaça à fauna de polinizadores nativos (Paton 1993; Huryn 1997), esta abelha parece não ser um problema para as espécies de Eugenia aqui estudadas, pois a polinização não é o principal fator limitante para a produção de frutos (comunicação pessoal, Silva \& Pinheiro).

Fenologia - Eugenia uniflora, E. neonitida, E. punicifolia e E. rotundifolia apresentam padrão de floração, em nível populacional, em massa e anual, com picos definidos de emissões florais. Cada indivíduo produz uma grande quantidade flores durante uma semana em média, porém há eventos episódicos de produção de algumas flores durante todo o ano. Segundo Wyk \& Lowrey (1988), Proença \& Gibbs (1994) e Lughadha \& Proença (1996) este padrão de floração é comum em muitas outras espécies de Eugenia.

Indivíduos de E. uniflora floresceram na estação seca do ano, de agosto a outubro, com frutificação de setembro a novembro e indivíduos E. neonitida floresceram do final de agosto até início de dezembro, com frutificação ocorrendo de outubro até início de janeiro. É evidente uma assincronia entre os indivíduos dentro das populações destas duas espécies, onde se podem encontrar plantas na fase reprodutiva ao mesmo tempo em que se encontram indivíduos na fase vegetativa. Esta característica pode aumentar as taxas de polinizações cruzadas dentro da população, pois promove uma maior amplitude de forrageio dos polinizadores em busca de alimento (Bawa 1983).

Eugenia punicifolia floresceu por duas vezes no ano de 2003, sendo a primeira florada entre os meses de junho e julho, frutificando no mês de agosto e a segunda, entre os meses de setembro e outubro, frutificando entre novembro e dezembro (Fig. 7). Já no ano de 2004, só ocorreu uma florada no mês de agosto com frutificação entre outubro e dezembro. Os indivíduos de E. rotundifolia floresceram na estação úmida do ano no mês de março, com frutificação de maio a junho (Fig. 7). Nestas duas espécies a floração é sincrônica dentro da população, pois a maioria dos indivíduos floresce ao mesmo tempo. Isto pode ter relação com o fato de que $E$. punicifolia e E. rotundifolia apresentam crescimento clonal e, portanto, os indivíduos de uma dada população são, na maioria, rametes interligados vegetativamente.

O padrão de floração das espécies aqui estudadas pode ser considerado, segundo Gentry (1974), como do tipo "big bang", pois há uma produção excessiva de flores em um curto período de tempo. A floração em massa individual e seqüencial garante, para os visitantes florais destas espécies, uma disponibilidade continua de grãos de pólen durante a fenofase de floração (Bawa 1983; Mori \& Pipoly 1984; Rathcke 1988;

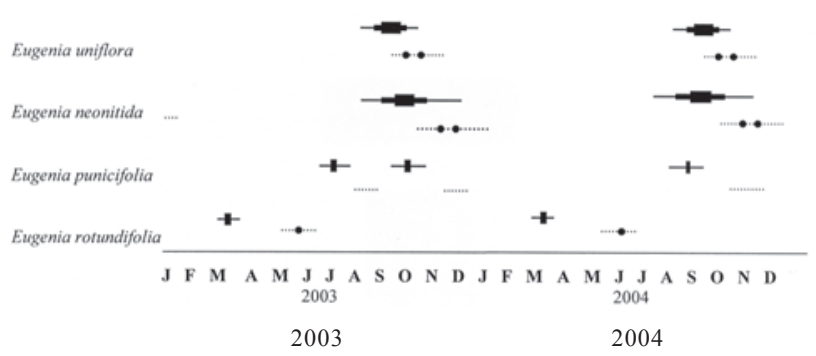

Figura 7. Período de floração (-) e de frutificação (.....) de espécies de Eugenia, em nível populacional, na restinga do Parque Natural Municipal de Grumari. A largura da linha e do tracejado significam aumento da intensidade do episódio de floração e de frutificação, respectivamente. 
Proença \& Gibbs 1994 e Lorenzon et al. 2003).

Eugenia uniflora, E. neonitida, E. punicifolia se enquadram nos dados encontrados por Proença \& Gibbs (1994) e Lughadha \& Proença (1996), em que a maioria das espécies de mirtáceas floresce, predominantemente, na transição entre a estação seca e úmida. No entanto, Gentry (1974) e Morellato \& Leitão Filho (1996), relataram que espécies com floração em massa, florescem na estação seca do ano. De fato, a floração ocorrendo na transição das estações seca e úmida, garante uma disponibilidade de frutos, principalmente, na estação úmida, que é a época de maior densidade de vertebrados dispersores de sementes na floresta atlântica (Tabarelli \& Peres 2002; Tabarelli et al. 2003). Além do mais, as sementes seriam dispersas antes do início da próxima estação seca, garantindo assim, a germinação e o estabelecimento das plântulas (Schaik et al. 1993; Proença \& Gibbs 1994; Morellato \& Leitão Filho 1996).

$\mathrm{Na}$ restinga de Grumari, os indivíduois de E. uniflora, E. neonitida, E. punicifolia e E. rotundifolia ocorrem muito próximos uns aos outros e como pode ser observado na Fig. 7, há uma evidente sobreposição na floração de E. uniflora, E. neonitida e E. punicifolia. Desta forma, os grãos de pólen destas espécies podem ser disponibilizados ao mesmo tempo para os insetos visitantes florais. Vários autores, entre eles Waser \& Real (1979), Ims (1990), Smith-Ramirez et al. (1998) e Barros (2002) defendem a "hipótese da facilitação", a qual se baseia na idéia de que a sobreposição na floração de espécies simpátricas poderia funcionar como um processo de mutualismo floral, aumentando assim a disponibilidade de recursos florais durante suas fenofases e, consequentemente, o número potencial de polinizadores e dispersores de sementes. Este processo pode funcionar bem em espécies que apresentem algum mecanismo de incompatibilidade interespecífica, que impediria a formação de híbridos (Waser \& Real 1979; Rathke 1988; Vogel \& Machado 1991; Arista et al. 1997). Por esta razão, se fazem necessários estudos mais detalhados sobre o fluxo gênico interespecífico entre as espécies de Eugenia na restinga de Grumari, mesmo não tendo sido observado no campo nenhum indivíduo com características intermediárias entre estas espécies. Dentre as quatro espécies de Eugenia deste estudo, apenas $E$. rotundifolia floresce em época diferente das demais, impossibilitando, desta forma, a transferência de pólen entre elas.

\section{Agradecimentos}

À CAPES, pela bolsa de Doutorado concedida ao primeiro autor; ao Programa de Pós-Graduação em Ciências Biológicas, Botânica do Museu Nacional, UFRJ, pelo incentivo a realização desta pesquisa; às Professoras Heloisa Alves de Lima, Lygia Fernandes e Maria Célia Correia Rodrigues (MN/UFRJ), pela leitura e sugestões dos manuscritos; à Secretaria Municipal de Meio Ambiente do Município do Rio de Janeiro, pela licença de pesquisa concedida na restinga do Parque Natural Municipal de Grumari; aos Professores Fernando Amaral da Silveira (UFMG), Gabriel Augusto Rogrigues de Melo (UFPR), Valéria Cid Maia (MN/UFRJ), Márcia Souto Couri (MN/UFRJ) e Miguel Angel Monné Barrios (MN/UFRJ), pela identificação dos insetos visitantes florais; à Professora Cleide Mendonça e aos Biólogos Eduardo e Liliane Henriques (MN/UFRJ), pelo apoio técnico.

\section{Referências bibliográficas}

Anderson, S.H. 2003. The relative importance of birds and insects as pollinators of the New Zeland flora. New Zeland Journal of Ecology 27(2): 83-94.

Aguiar, A.J.C. \& Martins, C.F. 2003. The bee diversity of the Tabuleiro vegetation in the Guaribas Biological Reserve (Mamanguape, Paraíba, Brazil). Pp. 209-216. In: G.A.R. Melo \& I. Alves-dos-Santos (eds.). Apoidea Neotropica: Homenagem aos 90 anos de Jesus Santiago Moure. Ed. Unesc.

Araújo, D.S.D. \& Henriques, R.P.B. 1984. Análise florística das restingas do Estado do Rio de Janeiro. Pp. 159-193. In: L.D. Lacerda; D.S.D. Araújo; R. Cerqueira \& B. Turcq (orgs.). Restingas: origem, estrutura, processos. Niterói, CEUFF .

Arista, M.; Ortiz, P.L. \& Talavera, S. 1997. Reproductive isolation of two sympatric subspecies of Juniperus phoenicea (Cupressaceae) in southern Spain. Plant Systematic and Evolution 208: 225-237.

Armstrong, J.A. 1979. Biotic pollination mechanisms in the Australian flora - A review. New Zealand Journal of Botany 17: 467-508.

Assis, A.M.; Thomaz, L.D. \& Pereira, O.J. 2004. Florística de um trecho de floresta de restinga no município de Guarapari, Espirito Santo, Brasil. Acta Botanica Brasilica 18(1): 191-201.

Barros, M.A.G. 2002. Floração sincrônica e sistemas reprodutivos em quatro espécies de Kielmeyera Mart. (Guttiferae). Acta Botanica Brasilica 16(1): 113-122.

Bawa, K.S. 1983. Patterns of flowering in tropical plants. Pp. 294-309. In: C.E. Jones \& R.J. Little (eds.). Handbook of Experimental Pollination biology. Van Nostrand Reinhold Company Inc. 
Beardsell, D.V.; O’Brien, S.P.; Williams, E.G.; Knox, R.B \& Calder, D.M. 1993. Reproductive biology of Australian Myrtaceae. Australian Journal of Botany 41: 511-526.

Briggs, B.G. \& Johnson, L.A.S. 1979. Evolution in the Myrtaceae - Evidence from inflorescence structure. Proceedings of the Linnean Society of New South Wales 102(4): 157-254.

Collevatti, R.G.; Schoereder, J.H. \& Campos, L.A.O. 2000. Foraging behavior of bee pollinators on the tropical weed Triumfetta semitriloba: Flight distance and directionality. Revista Brasileira de Biologia 60(1): 29-37.

Crome, F.H. \& Irvine, A.K. 1986. "Two Bob Each Way": The pollination and breeding system of the australian rain forest tree Syzygium cormiflorum (Myrtaceae). Biotropica 18(2): 115-125.

Darwin, C. 1859. A origem das espécies. Tradução Eugênio Amado. Belo Horizonte, Vila Rica ed. Grandes Obras da Cultura Universal.

Degenhardt, J.; Orth, A.I.; Guerra, M.P.; Ducroquet, J.P. \& Nodari, R.O. 2001. Morfologia floral da goiabeira serrana (Feijoa sellowiana) e suas implicações na polinização. Revista Brasileira de Frutificultura 23(3): 718-721.

Dobson, H.E.M. \& Bergström, G. 2000. The ecology and evolution of pollen odors. Plant Systematic and Evolution 222(1-4): 63-87.

Dreller, C. \& Tarpy, D.R. 2002. Perception of the pollen need by foragers in a honeybee colony. Animal Behaviour 59: 91-96.

Ducroquet, J.P.H.J. \& Hickel, E.R. 1997. Birds as pollinators of Feijoa (Acca sellowiana Bera). Acta Horticulture 452: 37-40.

Endress, P.K. 1994. Diversity and evolutionary biology of tropical flowers. Cambridge University Press.

Faegri, K. \& Pijl, L. van der. 1979. The principles of pollination ecology. Oxford, Pergamon Press.

Free, J.B. 1993. Insect pollination of crops. London, Academic Press.

Gentry, A.H. 1974. Flowering phenology and diversity in tropical Bignoniaceae. Biotropica 6(1): 64-68.

Gleeson, S.K. 1981. Character displacement in flowering phenologies. Oecologia 51: 294-295.

Grant, V. 1971. Plant Speciation. Ed. Columbia University Press.

Gross, C.L. \& Mackay, D. 1998. Honeybees reduce fitness in the pioneer shrub Melastoma affine (Melastomataceae). Biological Conservation 86: 169-178.

Heslop-Harrison, J. \& Shivanna, K.R. 1977. The receptive surface of the Angiosperm stigma. Annals of Botany 41(176): 1233-1258.

Hopper, S.D. 1980. Pollination of the rain-forest tree Syzygium tierneyanum (Myrtaceae) at Kuranda, Northen Queensland. Australian Journal of Botany 28: 223-237.

Huryn, V.M.B. 1997. Ecological impacts of introduced honey bees. The Quarterly Review of Biology 72(3): 275-297.

Ims, R.A. 1990. The ecology and evolution of reproductive synchrony. Tree 5(5): 135-140.

Johnson, L.A.S. \& Briggs, B.G. 1984. Myrtales and Myrtaceae - A phylogenetic approach. Annals of the Missouri Botanical Garden 71: 700-756.
Jones, G.D. \& Jones, S.D. 2001. The uses of pollen and its implication for entomology Neotropical. Entomology 30(3): 341-350.

Landroum, L.R. \& Kawasaki, M.L. 1997. The genera of Myrtaceae in Brazil: na illustrated synoptic treatment and identication keys. Brittonia 49(4): 508-536.

Larson, B.M.H.; Kevan, P.G. \& Inouye, D.W. 2001. Flies and flowers: taxonomic diversity of anthophiles and pollinators. The Canadian Entomologist 133: 439-465.

Latouche-Hallé, C.; Ramboer, A.; Bandou, E.; Caron, H. \& Kremer, A. 2004. Long-distance pollen flow and tolerance to selfing in a neotropical tree species. Molecular Ecology 13: 1055-1064.

Legrand, C.D. \& Klein, R.M. 1969. Flora Ilustrada Catarinense. Santa Catarina, Editor P. Raulino Reitz. I parte: Fascículo: Myrtaceae.

Lemos, M.C.; Pellens, R. \& Lemos, L.C. de 2001. Perfil e florística de dois trechos de mata litorânea no município de Maricá - RJ. Acta Botanica Brasilica 15(3): 321-334.

Lorenzon, M.C.A.; Matrangolo, C.A.R. \& Schoereder, J.H. 2003. Flora visitada pelas abelhas eussociais (Hymenoptera, Apidae) na Serra da Capivara, em Caatinga do sul do Piauí. Neotropical Entomology 32(1): 27-36.

Lughadha, E.N. \& Proença, C. 1996. A survey of the reproductive biology of the Myrtoideae (Myrtaceae). Annais of the Missouri Botanical Garden 83: 480-503.

Machado, I.C. \& Oliveira, P.E.A.M. 2000. Biologia floral e reprodutiva de Casearia grandiflora Camb. (Flacourtiaceae). Revista Brasileira de Botânica 23(3): 283-290.

Machado, I.C. \& Loiola, M.C. 2000. Fly pollination and pollinator sharing in two synchronopatric species: Cordia multispicata (Boraginaceae) and Borreria alata (Rubiaceae). Revista Brasileira de Botânica 23(3): 305-311.

Marchini, L.C.; Moreti, A.C.C.C.; Teixeira, E.W.; Silva, E.C.A.; Rodrigues, R.R. \& Souza, V.C. 2001. Plantas visitadas por abelhas africanizadas em duas localidades do Estado de São Paulo. Scientia Agricola 58(2): 413-420.

Maués, M.M. \& Couturier, G. 2002. Biologia floral e fenologia reprodutiva de camu-camu (Myrciaria dubia (H.B.K.) McVaugh, Myrtaceae) no Estado do Pará, Brasil. Revista Brasileira de Botânica 25(4): 441-448.

Merwe, M.M.; Wyk, A.E. \& Botha, A.M. 2004. Molecular phylogenetic analysis of Eugenia L. (Myrtaceae), with emphasis on southern Africa taxa. Plant Systematic Evolution. Published online: August 30, 2004.

Michener, C.D. 1979. Biogegraphy of the bees. Annals of the Missouri Botanical Garden 66: 277-347.

Moço, M.C.C. \& Pinheiro, M.C.B. 1999. Pollination ecology of Swartzia apelata Raddi var. apelata (Leguminosae Papilionioideae). Brazilian Archives of Biology and Technology 42(4): 415-423.

Mori, S.A. \& Pipoly, J.J. 1984. Observations on the big bang flowering of Miconia minutiflora (Melastomataceae). Brittonia 36(4): 337-341. 
Morellato, P.C. \& Leitão Filho, H.F. 1996. Reproductive phenology of climbers in a Southeastern Brazilian Forest. Biotropica 28(2): 180-191.

Nakagawa, M.; Itiotka, T.; Momose, K.; Yumoto, T.; Komai, F.; Morimoto, K.; Jordal, B.H.; Kato, M.; Kaliang, H.; Hamid, A.A.; Inoue, T. \& Nakashizuka, T. 2003. Resource use of insect seed predators during general flowering and seeding events in a Bornean dipterocarp rain forest. Bulletin of Entomological Research 93: 455-466.

O’Brien, S.P. \& Calder, D.M. 1993. Reproductive biology and floral phenologies of the sympatric species Leptospermum myrsinoides and L. continentale (Myrtaceae). Australian Journal of Botanic 41: 527-539.

Paton, D.C. 1993. Honeybees in the australian environment. Does Apis mellifera disrupt or benefit the native biota? Bioscience 43(2): 95-103.

Pernal, S.F. \& Currie, R.W. 2002. Discrimination and preferences for pollen-based cues by foraging honeybees, Apis mellifera L. Animal Behaviour 63: 369-390.

Peters, C.M. \& Vasquez, A. 1986/1987. Estudios ecológicos de Camu-camu (Myrciaria dubia). I. Produccion de frutos en poblaciones naturales. Acta Amazonica 16/17: 161-174.

Proctor, M. \& Yeo, P. 1973. The pollination of flowers. London, Collins.

Proença, C. 1992. Buzz pollination - older and more widespread than we think? Journal of Tropical Ecology 8: $115-120$.

Proença, C. \& Gibbs, P.E. 1994. Reproductive biology of eight sympatric Mirtaceae from Central Brazil. New Phytologisty 126: 343-354.

Ramalho, M.; Kleinert-Giovannini, A. \& Imperatriz-Fonseca, V.L. 1990. Important bee plants for stingless bees (Melipona and Trigonini) and africanized heneybees (Apis mellifera) in neotropical habitats: a review. Apidologie 21: 469-488.

Ramalho, M. 2003. Stingless bees and mass flowering trees in the canopy of Atlantic forest: a tight relationship. Acta Botanica Brasilica 18(1): 37-47.

Rathcke, B. 1988. Interactions for pollination among coflowering shrubs. Ecology 69(2): 446-457.

Roitman, G.G.; Montaldo, N.H. \& Medan, D. 1997. Pollination biology of Myrrhinium atropurpureum (Myrtaceae): Sweet, flesh petals attract frugivorous birds. Biotropica 29(2): 162-168.

Roulston, T.H. \& Cane, J.H. 2000. Pollen nutritional content and digestibility for animals. Plant Systematic and Evolution 222(1-4): 187-209.

Sazima, M. 1978. Polinização por moscas em Bulbophylum warmingianum Cogn. (Orchidaceae), na Serra do Cipó, Minas Gerais, Brasil. Revista Brasileira de Botânica 1:133-138.

Schaik, C.P.; Terborgh, J.W. \& Wright, S.J. 1993. The phenology of tropical forests: Adaptive significance and consequences for primary consumers. Annual Review of Ecology and Systematics 24: 353-377.
Silva, A.L.G.; Ormond, W.T. \& Pinheiro, M.C.B. 2002. Biologia floral e reprodutiva de Senna australis (Vell.) Irwin \& Barneby (Fabaceae - Caesalpinioideae). Boletim do Museu Nacional (N. S. Bot.) 121: 1-11.

Siqueira de Castro, M. 2002. Bee fauna of some tropical and exotic fruits: Potencial pollinators and their conservation. Pp. 275-288. In: P. Kevan \& V.L. Fonseca Imperatriz (eds.). Pollinating bees - The conservation link between agriculture and nature-Ministry of Environment, Brasília.

Smith-Ramirez, C.; Armesto, J.J. \& Figuerosa, J. 1998. Flowering, fruiting and seed germination in Chilean rain forest Myrtaceae: ecological and phylogenetic constraints. Plant Ecology 136: 119-131.

Stebbins, G.L. 1950. Variation and Evolution in Plants. Ed. Columbia University Press.

Taberelli, M. \& Peres, C.A. 2002. Abiotic and vertebrate seed dispersal in the Brazilian Atlantic forest: implications for forest regeneration. Biological Conservation 106: 165-176.

Taberelli, M.; Vicente, A. \& Barbosa, D.C.A. 2003. Variation of seed dispersal spectrum of woody plants across a rainfall gradient in north-eastern Brazil. Journal of Arid Environments 53: 197-210.

Taylor, G. \& Whelan, R.J. 1988. Can honeybees pollinate Grevillea? Australian Zoologist 24(4): 193-196.

Thorp, R.W. 2000. The collection of pollen by bees. Plant Systematic and Evolution 222(1-4): 211-223.

Vaughton, G. 1996. Pollination disruption by European honeybees in the Australian bird-pollinated shrub Grevillea barklyna (Proteaceae). Plant Systematic and Evolution 200: 89-100.

Villanueva-G., R. 2002. Polliniferous plants and foraging strategies of Apis mellifera (Hymenoptera: Apidae) in the Yacatán Peninsula, México. Revista de Biologia Tropical 50(3/4): 1035-1044.

Vogel, S.T. 1978. Evolutionary shifts from reward to deception in pollen flowers. In: A.J Richards (ed.). The pollination of flowers by insects. Linnean Society Symposium Series 6: 89-96.

Vogel, S.T. 1990. The role of scent glands in pollination. Washington, D.C., The National Science Foundation.

Vogel, S.T. \& Machado, I.C. 1991. Pollination of four sympatric species of Angelonia (Scrophulariaceae) by oil-collecting bees in NE. Brazil. Plant Systematic and Evolution 178: 153-178.

Waser, N.M. \& Real, L.A. 1979. Effective mutualism between sequentially flowering plant species. Nature 281(25): 670-672.

Wilson, G.W.; O’Brien, M.M.; Gadek, P.A. \& Quinn, C.J. 2001. Myrtaceae revisited: A reassessment of intrafamilial groups. American Journal of Botany 88(11): 2013-2025.

Wyk, A.E. \& Lowrey, T.K. 1988. Studies on the reproductive biology of Eugenia L. (Myrtaceae) in Southern Africa. Monography Systematic Botanic Missouri Botanical Garden 25: 279-293. 